\title{
ON ALL KINDS OF HOMOGENEOUS SPACES
}

\author{
BY
}

\author{
GERALD S. UNGAR
}

ABSTRACT. Several open questions on homogeneous spaces are answered. A few of the results are:

(1) An $n$-homogeneous metric continuum, which is not the circle, is strongly $n$-homogeneous.

(2) A 2-homogeneous metric continuum is locally connected.

(3) If $X$ is a homogeneous compact metric space or a homogeneous locally compact, locally connected separable metric space, then $X$ is a coset space.

(4) If $G$ is a complete separable metric topological group with is $n$-connected, then $G$ is locally $n$-connected.

1. Introduction. At the topology conference at the University of Wisconsin in 1955 Burgess [3] asked the following questions.

(1) Does there exist a continuum different from a simple closed curve which, for some positive integer $n$, is $n$-homogeneous but not strongly $n$-homogeneous?

(2) Is every 2-homogeneous compact metric continuum locally connected?

(3) Are there continua for which to each pair of their points there exists a unique homeomorphism carrying one of the points onto the other?

The paper will give almost complete answers to all of these questions in the metric case (although only (2) mentions the word metric, all of Burgess' work on homogeneity is on metric spaces). The answer to (1) will be no. The answer to (2) will be yes. It will also be shown that a strong type of 1-homogeneity gives an affirmative answer to (2). It seems like the answer to (3) should be no. It is shown that if a space satisfies the condition of (3) then it is an abelian topological group and applying structure theorems in [9], it is shown that it cannot be finite dimensional.

The main tool of this paper is Theorem 2.1 of [5]. This theorem gives a condition for a space to be a coset space of a topological group, and as a direct result of it, we can prove that compact metric homogeneous spaces and locally compact locally connected separable metric spaces are cosets spaces of their group of homeomorphisms with the $C-0$ topology. This answers questions raised by the work of Ford [6], Mostert [10] and Ungar [12]. As a matter of fact in these last three references, two types of homogeneity were defined in order to

Received by the editors September 20, 1974.

AMS (MOS) subject classifications (1970). Primary 54F99; Secondary 54H15. 
guarantee that certain homogeneous spaces were coset spaces and yet none of the above werc able to show, as $\mathrm{Wu}[16]$ did, that the pseudo arc is a coset space.

As a consequence of Effros' theorem we will also give a proof of a theorem which states that a complete separable metric topological group which is $n$-connected is locally $n$-connected. This overlaps the work of Rickert [11] for the case $n=0$.

2. Definitions and notations.

(2.1). Notation. Let $X$ be a topological space.

(a) $F_{n}(X)$, the nth configuration space of $X$, will be $X^{n}-\left\{\left(x_{1}, \ldots, x_{n}\right)\right.$ $\in X^{n} \mid x_{i}=x_{j}$ for some $\left.i \neq j\right\}$.

(b) $H(X)$ will denote the group of homeomorphisms of $X$ onto itself. If we refer to $H(X)$ as a topological space we will assume that it has the compact open topology.

(c) $H_{x}=\{h \in H(X) \mid h(x)=x\}$.

(d) $B^{k+1}$ will be the unit $k+1$ ball in $k+1$ space and $S^{k}$ will be its boundary.

(2.2). Definition. A space $X$ is $n$-homogeneous [strongly n-homogeneous] if, given any two points $\left(x_{1}, \ldots, x_{n}\right)$ and $\left(y_{1}, \ldots, y_{n}\right)$ of $F_{n}(X)$, there exists a homeomorphism $h$ of $X$ onto itself such that $h\left(\bigcup x_{i}\right)=\bigcup y_{i}\left[h\left(x_{i}\right)=y_{i}\right]$. A space is homogeneous if it is 1-homogeneous.

(2.3). Definition. A topological transformation group $(G, X)$ is a topological group $G$ together with a topological space $X$ and a continuous map $(g, x)$ $\rightarrow g x$ of $G \times X$ into $X$ such that $(g h) x=g(h x)$. A topological transformation group $(G, X)$ is polonais (polish) if both $G$ and $X$ are polonais, i.e. they are separable and metrizable by a complete metric. If $(G, X)$ is a topological transformation group and $x \in X$, let $G_{x}$ be the isotropy subgroup of $G$, i.e. $\{g \in G \mid g x=x\}$. A topological transformation group is transitive if for every two points $x$ and $y$ of $X$ there exists $g \in G$ such that $g x=y$.

(2.4). REMARKS. (a) Locally compact separable metric spaces are polonais.

(b) $H(X)$ acts on $F^{n}(X)$ as follows: $h_{1}\left(x_{1}, \ldots, x_{n}\right) \rightarrow\left(h\left(x_{i}\right), \ldots\right.$, $\left.h\left(x_{n}\right)\right)$ and if we refer to $H(X)$ acting on it, this action will be understood.

(c) $X$ is strongly $n$-homogeneous iff $H(X)$ acts transitively on $F^{n}(X)$.

(2.5). Definition. A topology $\tau$ for $H(X)$ will be reasonable if $((H(X), \tau), X)$ is a topological transformation group and the map $\phi_{x}: H(X) / H_{x}$ $\rightarrow X$ defined by $\phi_{x}: g H_{x} \rightarrow g(x)$ is a homeomorphism. Note that for $H(X)$ to have a reasonable topology it is necessary that $X$ be homogeneous.

(2.6). Definition. A space $X$ is $C^{n}$ iff any map $f: S^{k} \rightarrow X\{k \leqslant n\}$ has an extension from $B^{k+1}$ into $X$. It is $L C^{n}$ if given any point $x \in X$ and any 
neighborhood $U$ of $X$ there exists a neighborhood $V$ of $x$ such that any map $f$ : $S^{k} \rightarrow V, k \leqslant n$, has an extension from $B^{k+1}$ into $U$.

Note $L C^{0}$ is the same as locally arcwise connected.

(2.7). Conventions. (1) Map = continuous function.

(2) Continuum $=$ compact connected Hausdorff space.

3. Everything else. We are now in a position to state Theorem 2.1 of [5] Let $(G, X)$ be a polonais transformation group. Then the following are equivalent.

(1) For each $x$ in $X$ the map $g G_{x} \rightarrow g x$ of $G / G_{x}$ onto $G x$ is a homeomorphism.

(2) Each orbit (i.e. $G x$ ) is a $G_{\delta}$ in $X$.

If $(G, X)$ is transitive then the orbits are all of $X$ and hence certainly $G_{\delta}$ 's in $X$. Hence we have

(3.1). THEOREM. If $(G, X)$ is a transitive polonais transformation group then for each $x$ in $X$ the map $\phi_{x}: g G_{x} \rightarrow g x$ of $G / G_{x}$ onto $X$ is a homeomorphism and hence the map $T_{x}: g \rightarrow g x$ of $G$ onto $X$ is an open map.

The above will be clearer if we note that the following diagram commutes and that the natural map $G \rightarrow G / G_{x}$ is always an open map.

$$
\underset{G / G_{x}}{\stackrel{T_{x}}{\longrightarrow} X}
$$

Before answering Burgess' questions, we will prove the results mentioned in the last half of the Introduction.

(3.2). THEOREM. If $X$ is a homogeneous compact metric space, then the C - 0 topology is reasonable for $H(X)$.

Proof. This follows from (3.1) because $H(X)$ with $C-0$ topology has a complete metric and is a separable topological group acting continuously on $X$.

(3.3). THEOREM. If $X$ is a homogeneous locally compact locally connected separable metric space, then the $C-0$ topology is reasonable for $H(X)$.

Proof. Let $\hat{X}$ be the one point compactification of $X$. $\hat{X}$ is compact metric, hence $H(\hat{X})=\hat{H}$ is separable complete metric and $H(X)$ is homeomorphic to $\hat{H}_{x}$ [2] which is closed in $\hat{H}$. Therefore $H(X)$ with the $C-0$ topology is separable complete metric and hence we could apply (3.1) to get our result.

Before proving the next theorem we need the following "well-known" lemmas. 

$x$ in $X$.

(3.4). Lemma [15]. A space $X$ is $L C^{n}$ iff $(X, x)^{\left(I^{n}, S^{n-1}\right)}$ is $L C^{0}$ for all

(3.5). Lemma [14]. An arcwise connected space $X$ is $L C^{0}$ iff the map $F$ : $X^{I} \rightarrow X \times X$, defined by $F(\alpha)=(\alpha(0), \alpha(1))$, is open.

(3.6). LemMA [1]. If $G$ is a topological group and $X$ is locally compact, then $G^{X}$ is a topological group with multiplication defined by $\alpha \cdot \beta(x)=\alpha(x)$. $\beta(x)$.

(3.7). Theorem. Let $G$ be a $C^{n}$ connected separable complete metric group, then $G$ is $L C^{n}$.

Proof. By (3.5) we need only show that if $X=(G, e)^{\left(I^{n}, s^{n-1}\right)}$ then the map $F: X^{I} \rightarrow X \times X$, defined by $F(\alpha)=(\alpha(0), \alpha(1))$, is open. However, by (3.6), $X$ and $X^{I}$ are topological groups which are complete separable metrics and it is easily seen that $F$ is a homomorphism. Hence by [8, Corollary $6, \S 2]$ or by noting that homomorphisms induce group actions and applying (3.1) we get that $F$ is open as desired.

Now we will give the answers to Burgess' questions.

(3.8). THEOREM. If $X$ is an n-homogeneous compact metric space or locally compact locally connected separable metric space such that $F^{n}(X)$ is connected, then $X$ is strongly n-homogeneous.

Proof. In both cases $H(X)$ is a complete separable metric topological group. Let $G$ be the symmetric group on $\{1, \ldots, n\}$. If $G$ has the discrete topology, then $H(X) \times G$ is a complete separable metric topological group with multiplication defined by $\left(h_{1}, g_{1}\right) \cdot\left(h_{2}, g_{2}\right)=\left(h_{1} h_{2}, g_{2} g_{1}\right)$. Define an action of $H(X) \times$ $G$ on $F^{n}(X)$ as follows:

$$
(h, \pi)\left(x_{1}, \ldots, x_{n}\right) \rightarrow\left(h\left(x_{\pi(1)}\right), \ldots, h\left(x_{\pi(n)}\right)\right) .
$$

It is easily seen that this is a continuous action. If $X$ is $n$-homogeneous, then this action is transitive. Hence, by (3.1), $T_{\left(x_{1}, \ldots, x_{n}\right)}: H(X) \times G \rightarrow F^{n}(X)$ is an open map for every $\left(x_{1}, \ldots, x_{n}\right) \in F^{n}(X)$. If $g \in G$ we then have that $\left.T_{\left(x_{1}, \ldots, x_{n}\right)}(H(X) \times g)\right)$ is open in $F^{n}(X)$. For simplicity call this set $A g$. Since the above action is transitive, $\bigcup A g=F^{n}(X)$. However, $A g$ is an orbit of the action of $H(X)$ on $F_{n}(X)$. That is, $A g=H(X)\left(x_{g(1)}, \ldots, x_{g(n)}\right)$. However, orbits are either disjoint or equal and since $F_{n}(X)$ is connected, all of the orbits must be equal to $F_{n}(X)$. Therefore $X$ is strongly $n$-homogeneous.

(3.9). Lemma. $F_{n}(X)$ is connected if no set of $n-1$ points separate $X$.

Proof. The proof will be by induction. $F_{1}(X)=X$ is connected iff the 
empty set does not separate $X$. Assume the theorem is true for $n=k$. Consider the case for $n=k+1$. Let $\pi: F_{k+1}(X) \rightarrow F_{k}(X)$ be the projection on the first $k$ coordinates $\pi^{-1}\left(a_{1}, \ldots, a_{k}\right)=a_{1} \times \cdots \times a_{k} \times\left(X-\bigcup_{i=1}^{k} a_{i}\right)$. If no $k$ points separate $X$, then $\pi^{-1}\left(a_{1}, \ldots, a_{k}\right)$ is connected. The map $\pi$ is open and by induction $F_{k}(X)$ is connected. An elementary theorem in topology states if $f$ is an open map from a space $Y$ onto a connected space $Z$ such that each point inverse is connected, then $Y$ is connected. From the above we have that $\pi$ satisfies the conditions of this theorem and hence $F_{k+1}(X)$ is connected.

(3.10). Corollary. If $X$ is an n-homogeneous compact metric space or locally compact locally connected separable metric space such that no set of $n-1$ points separates $X$, then $X$ is strongly n-homogeneous.

(3.11). THEOREM. If $X$ is an n-homogeneous compact metric continuum, then $X$ is strongly n-homogeneous or $X$ is the circle.

Proof. The theorem is obviously true for $n=1$. Hence if $n \geqslant 2$ we have either that no set of $n-1 \geqslant 1$ points separates $X$ and hence by (3.10), $X$ is strongly $n$-homogeneous or some set of $n-1$ points separates $X$. However, [4, Theorem 13] states that if a homogeneous compact metric continuum is separated by some countable set, then it is a circle. Hence the proof is complete.

(3.12). THEOREM. If $X$ is a 2-homogeneous metric continuum then $X$ is locally connected.

Proof. By (3.11) we will assume that $X$ is strongly 2-homogeneous. Again we will set things up so that we could use (3.1).

Since $X$ is compact metric, $H(X)$ is complete metric and $H_{x}$ is closed in $H(X)$ for each $x$ in $X$, therefore $H_{x}$ is also complete metric. Since $X$ is strongly 2-homogeneous $H_{x}$ acts transitively on $X-x$. We now have the hypothesis of (3.1) satisfied. Since $\left(H_{x}, X-x\right)$ is a transitive topological transformation group, $H_{x}$ is complete separable metric and $X-x$ is locally compact metric, hence complete metric. Therefore, we have that, for any $y \in X-x$, the map $T_{y}: H_{x}$ $\rightarrow X-x$ defined by $T_{y}(g)=g(y)$ is open.

The openness of $T$ at the identity is equivalent to the following condition.

(1) Given any two points $x$ and $y$ of $X$ and any $\epsilon>0$ there exists $\delta>0$ such that if $z \in N(y, \delta)$ then there exists a homeomorphism $h$ such that $h(x)=$ $x, h(y)=z$ and $d(h, i d)<\epsilon(d$ is the sup metric on $H(X))$. Note that although $d$ may not be the complete metric used in (3.1) the hypothesis yields that the $d$ topology is the same as a topology generated by a complete metric.

We see that (1) is true since $T_{y}\left(N_{\epsilon}(i d)\right)$ is open in $X-x$.

In order to complete the proof let $U$ be open in $X$ and let $C$ be a component 
of $U$ and let $y \in C$. There exists $\epsilon>0$ such that $N(y, 4 \epsilon) \subset U$. Let $X$ be a point different from $y$ such that $x$ is in the same component of $N(x, \epsilon)$ which contains $z$. (It is easy to show that such points exist.) Now by condition (1) there exists $\delta>0$ such that, if $z \in N(y, \delta)$, then there exists a homeomorphism $h$ such that $h(x)=x, h(y)=z$ and $d(h, i d)<\epsilon$. I claim that $N(y, \delta) \subset C$. This is easy to see since $x$ and $y$ are in a connected set of diameter less than $2 \epsilon$ and if $z \in N(y, \delta)$ the homeomorphism given by (1) takes this connected set to one which contains $x$ and $z$ and no point gets moved more than $t$. Hence $x$ and $z$ are in a connected set in $N(z, 4 \epsilon)$. Therefore $x, y$ and $z$ are all in the same component of $U$ as desired.

(3.13). Definition. A space $X$ is homeotopically homogeneous if given any two points $x$ and $y$ of $X$ there exists a homeomorphism $h$ of $X$ onto itself which takes $x$ to $y$ and which is homeotopic to the identity.

(3.14). THEOREM. If $X$ is a homeotopic homogeneous metric continuum, then $X$ is locally connected.

Proof. By $[13,(3.5)], X \times X$ is strongly $n$-homogeneous for all $n$. Hence, by (3.12), $X \times X$ is locally connected, which implies that $X$ is locally connected.

Finally, we come to question (3) of Burgess. A partial answer will follow from the next theorem which is another application of (3.1).

(3.15). THEOREM. Let $X$ be either a compact metric space or a locally compact, locally connected, separable metric space such that for every pair of points in $X$ there is a unique homeomorphism of $X$ onto itself which takes one point to the other. Then $H(X)$ is homeomorphic to $X$.

Proof. Again $H(X)$ satisfies the hypothesis of (3.1). Hence, for each $x$, $T_{x}: H(X) \rightarrow X$ is open. However, the hypothesis of the theorem also implies that $T_{x}$ is one-to-one and onto. Therefore $T_{x}$ is a homeomorphism.

(3.16). THEOREM. If $X$ satisfies the hypothesis of (3.15) then $X$ is an abelian topological group and the group of topological automorphisms of $X$ is trivial (i.e. consists of just the identity).

Proof. That $X$ has the structure of a topological group follows from (3.15). If we let $L_{x}: X \rightarrow X$ be defined by $L_{x}(y)=x y$, then we see that, for any $x$ and $y, L_{y x^{-1}}$ is the unique homeomorphism which takes $x$ to $y$. For any $x$ define $I_{x}: X \rightarrow X$ by $I_{x}(y)=x^{-1} y x$. Then $I_{x}$ is a homeomorphism of $X$ onto itself and $I_{x}(e)=e$. Therefore $I_{x}$ is the identity which is $L_{e}$. Therefore $x^{-1} y x=y$ for all $x$ and $y$ and hence $C$ is abelian. Finally if $A$ is any automorphism of $X$ again we have $A(e)=e$ so $A$ must be the identity. 
(3.17). THEOREM. There are no finite dimensional spaces satisfying the hypothesis of (3.15).

Proof. If $X$ is a locally compact locally connected separable metric finite dimensional space which satisfies the hypothesis of (3.1) then, by (3.16), $X$ is a topological group and hence by $[9$, Theorem 4.10 .1$], X$ is a Lie group and one can then show it has a lot of homeomorphisms.

If $X$ is just compact metric and connected then, by [9, Theorem 4.9.3], $X$ is locally the product of a compact totally disconnected group and a Euclidean neighborhood and again one can show it has a lot of homeomorphisms.

Note that (3.17) does not use the fact that $X$ is abelian or that the automorphism group is trivial. I feel that there must be a theorem somewhere in the literature that would handle the infinite dimensional case.

(3.18). Interesting trivia. (1) A compact group $G$ cannot act 2 transitively on a space unless $F_{2}(X)$ is compact. This is hardly ever the case.

Proof. If $G$ acts 2-transitively on $X$, then it acts transitively on $F_{2}(X)$. Hence the orbits which are compact must be $F_{2}(X)$.

(2) If a space is 2-homogeneous and admits a nontrivial involution then it is strongly 2-homogeneous.

Proof. Let $\alpha$ be the nontrivial involution and let $\alpha(a)=b$ so that $\alpha(b)=$ a. Given $x_{1}, x_{2}, x_{1} \neq x_{2}$. By the 2-homogeneity there exists a homeomorphism $h$ such that $h\left(x_{1} \cup x_{2}\right)=(a \cup b)$. If $h\left(x_{1}\right)=a$ and $h\left(x_{2}\right)=b$, that is okay; if not, then $\alpha h\left(x_{1}\right)=a$ and $\alpha h\left(x_{2}\right)=b$. Hence we have shown given any two points $x_{1}, x_{2}$ there exists a homeomorphism $g$ such that $g\left(x_{1}\right)=a$ and $g\left(x_{2}\right)=b$. This is sufficient for the strong 2-homogeneity of $X$.

(3) If $X \times X$ is 2-homogeneous, then it is strongly 2-homogeneous.

Proof. This follows from (2) since $X \times X$ admits a nontrivial involution.

(4) If $X$ is 2-homogeneous but not strongly 2-homogeneous, then $H(X)$ contains no elements of even order.

Proof. This is just like (2).

\section{REFERENCES}

1. R. F. Arens, A topology for spaces of transformations, Ann. of Math. (2) 47 (1946), 480-495. MR 8, 165.

610. MR 8, 479.

2.

3. C. E. Burgess, Homogeneous continua, Summary of Lectures and Seminars, Summer Institute on Set Theoretic Topology, University of Wisconsin, 1955, pp. 75-78.

4. - Continua and various types of homogeneity, Trans. Amer. Math. Soc. 88 (1958), 366-374. MR 20 \#1961.

5. E. G. Effros, Transformation groups and C-algebras, Ann. of Math. (2) 81 (1965), 38-55. MR 30 \#5175. 
6. L. R. Ford, Jr., Homeomorphism groups and coset spaces, Trans. Amer. Math. Soc. 77 (1954), 490-497. MR 16, 609.

7. J. G. Hocking and G. S. Young, Topology, Addison-Wesley, Reading, Mass., 1961. MR 23 \#A2857.

8. T. Husain, Introduction to topological groups, Saunders, Philadelphia, Pa., 1966. MR 34 \#278.

9. D. Montgomery and L. Zippin, Topological transformation groups, Interscience, New York and London, 1955. MR 17, 383.

10. P. S. Mostert, Reasonable topologies for homeomorphism groups, Proc. Amer. Math. Soc. 12 (1961), 598-602. MR 24 \#A541.

11. N. W. Rickert, Some properties of locally compact groups, J. Austral. Math. Soc. 7 (1967), 433-454. MR 36 \#2735.

12. G. S. Ungar, Local homogeneity, Duke Math. J. 34 (1967), 693-700. MR 36 \#869.

13. - Products and n-homogeneity (to appear).

14. - Relations between the covering homotopy and slicing structure properties, Illinois J. Math. 18 (1974), 236-245.

15. Completely regular maps, fiber maps and local n-connectivity, Proc. Amer. Math. Soc. 21 (1969), 104-108. MR 39 \#7601.

16. T.S. Wu, Each homogeneous nondegenerate chainable continuum is a coset space, Proc. Amer. Math. Soc. 12 (1961), 843-845. MR 23 \#A3801.

DEPARTMENT OF MATHEMATICS, UNIVERSITY OF CINCINNATI, CINCINNATI, OHIO 45221 